\title{
Diagnostic and Prognostic Value of Neutrophil Lymphocyte Ratio in Patients with Acute Pancreatitis
}

\author{
(D) Jale Memmedova1, (D) Yasemin Gökden², (D) Mine Adaş3 \\ 1istanbul Okmeydanı Training and Research Hospital, Clinic of Internal Medicine, istanbul, Turkey \\ 2 istanbul Okmeydanı Training and Research Hospital, Clinic of Gastroenterology, Istanbul, Turkey \\ 3 istanbul Okmeydanı Training and Research Hospital, Clinic of Endocrinology, Istanbul, Turkey
}

\section{Abstract}

Objective: Acute pancreatitis is an inflammatory disease of the pancreas with local and systemic complications, and high mortality and morbidity. A variety of scoring systems have been developed for the early detection of severe acute pancreatitis. However, they are not feasible enough to detect severe patients in the early period. This study aimed to investigate the relationship between neutrophil lymphocyte ratio (NLR) and etiology of acute pancreatitis, clinical presentation, early diagnosis of severe pancreatitis, length of hospital stay and complications.

Methods: A total of 150 patients with acute pancreatitis and 36 age and gender matched healthy controls were included. NLR was calculated within the first 24 hours of admission to the hospital. The results were compared with disease etiology, activity indices, complications, and length of hospital stay.

Results: In patients with acute pancreatitis, NLR levels $(7.0 \pm 9.3)$ were found to be significantly higher than in the control group (1.8 \pm 0.6$)(p<0.05)$. There was no statistically significant difference between biliary and non-biliary pancreatitis groups in terms of neutrophil, lymphocyte, NLR, CRP and sedimentation levels ( $p>0.05$ ). Regarding Ranson's criteria and Modified Glasgow Prognostic score, NLR was significantly higher in patients with severe pancreatitis $(p<0.05)$. However, there was no similar correlation with Balthazar score.

Conclusion: NLR was shown to increase in patients with acute pancreatitis as well as in other inflammatory conditions. NLR may be useful as an easily applicable parameter in the early diagnosis of disease and prediction of disease activity in patients with acute pancreatitis.

Keywords: Acute pancreatitis, neutrophil, lymphocyte, neutrophil lymphocyte ratio

\section{INTRODUCTION}

Acute pancreatitis (AP) is an acute inflammatory disease of the pancreas that develops when inactive pancreatic enzymes in the pancreatic parenchyma become active for various reasons or when the activated enzymes are not sufficiently inactivated (1). AP is an important disease that causes morbidity and mortality. The annual incidence ranges from 4.9 to 35 per 100.000 (2). In the United State, more than 300.000 patients are diagnosed with AP annually and approximately 20.000 patients die (3). Eighty percent of the cases are mild and recover without serious morbidity, but $20 \%$ have a severe course. There was no decrease in the frequency of severe pancreatitis over time (4). Different scoring systems have been developed to assess the severity of AP based on clinical and laboratory findings, radiological risk factors, severity grading systems, and various serum markers. Some of these scoring systems are used in the first hours of admission, while others are used in the first 48-72 hours or later. However, these predictive models have low specificity due to high false positive rates (5). Since the physiological response of circulating leukocytes to stress causes an increase in the number of neutrophils and a decrease in the number of lymphocytes, the ratio of these two subgroups is used as a marker of inflammation in inflammatory diseases (6). Severe AP is said to lead to poor prognosis, uncontrolled Systemic Inflammatory Response syndrome (SIRS) and Multiple Organ Dysfunction syndrome. White blood cell (WBC) count is one of the criteria for SIRS score in AP (7). The decreased number of lymphocytes associated with an increased number of neutrophils is associated with severe 
sepsis, bacteremia and surgical stress. Neutrophils trigger SIRS and inflammatory cascade, while lymphocytes decrease in severe sepsis. It has been associated with adverse outcomes in patients $(6,8,9)$. The aim of this study was to investigate the relationship between neutrophil lymphocyte ratio (NLR) and etiology of AP, clinical presentation, early diagnosis of severe pancreatitis, length of hospital stay and complications.

\section{METHODS}

The study was approved by the Ethics Committee with decision no. 481, dated 17.05.2016.

The study included 150 patients who were older than 18 years, who had no history of malignant disease, and who were clinically and radiologically diagnosed with AP and hospitalized to the internal medicine clinic between 01.03.2014 and 01.04.2016. The files of the patients were scanned from the Hospital Information Management System. The results of radiological examinations were retrospectively analyzed in terms of age, gender, length of hospital stay, complete blood count, biochemistry, C-reactif protein (CRP) and sedimentation rate. We investigated the relationship between NLR in the first 24 hours after admission and etiology, as well as the relationship between NLR and clinical parameters [Ranson's criteria and Modified Glasgow Prognostic Score (mGPS)], inflammation parameters (CRP, sedimentation), radiological grading (Balthazar score) and length of hospital stay. The severity of pancreatitis was accepted as mild (0-3 points) and severe (4-11 points) according to Ranson's criteria. According to mGPS, score $\geq 3$ was considered as severe pancreatitis $(10,11)$. In the scales defined by Balthazar et al. (12) the grading was based on the degree of necrosis, the presence of inflammation and fluid collection. It was classified as mild (0-3 points), moderate (4-6 points) or severe (7-10 points) (12). Thirty six gender- and age-matched healthy individuals were included in the study as the control group.

\section{Statistical Analysis}

Statistical analysis was performed using SPSS 22.0. In descriptive statistics, categorical data were expressed as number and percentage, and continuous data as mean, standard deviation, median, maximum and minimum. Normality tests were carried out using Kolmogorov-Smirnov test. The comparison of the continuous data from two independent groups was made using Independent Samples t-test if the assumptions of normality were met, and Mann-Whitney $U$ test if not. Kruskall-Wallis test was used to compare continuous data in which a maximum of two independent groups were provided with the assumptions of non-normality. If differences were found between the groups, the difference is determined by adjusting the $p$ values. Chi-square test was used to analyze categorical variables and Fisher's Exact test was used as the alternative when chi-square test conditions were not met. Spearman correlation was used for correlation analysis. The effect level and cut-off value were investigated by ROC curve.

\section{RESULTS}

A total of 150 patients with AP and 36 healthy individuals who met the inclusion criteria were included. Seventy-five AP patients (50\%) were female and 75 (50\%) were male. Of controls, $12(33.3 \%)$ were male and 24 (66.7\%) were female. The mean age of the patient group was $54.1 \pm 18.2$ years and the mean age of the control group was $49.1 \pm 12.4$ years. There was no statistically significant difference between the two groups in terms of age and gender ( $p>0.05$, Table 1). The etiology of 150 AP cases was biliary in $41 \%(n=61)$ and non-biliary (alcohol, hypertriglyceridemia, drugs, autoimmunity, trauma, idiopathic) in $59 \%(n=89)$. The Ranson's criteria score at 48 hours was between $0-3$ points (mild AP) in 121 patients (80.7\%) and between 4-11 points (severe AP) in 29 patients (19.3\%). According to mGPS within 48 hours after admission, $82 \%$ of the cases were evaluated as mild AP and $18 \%$ as severe AP. When the patient and control groups were compared in terms of neutrophil and lymphocyte levels in the first 24 hours after hospital admission, patient group had higher neutrophil levels and lower lymphocyte levels than the control group $(p<0.005)$. The mean NLR was $7.0 \pm 9.3$ in the patient group and $1.8 \pm 0.6$ in the control group. NLR was significantly higher in the patient group than the control group $(p<0.05$, Table 1$)$. The diagnostic value of NLR in distinguishing AP from the control group was investigated by plotting a ROC curve. It was found to

Table 1. Demographic characteristics and laboratory analysis of patients and controls

\begin{tabular}{|l|l|l|l|}
\hline & $\begin{array}{l}\text { Patients } \\
\text { with acute } \\
\text { pancreatitis }\end{array}$ & $\begin{array}{l}\text { Healthy } \\
\text { controls }\end{array}$ & $\mathbf{p}$ \\
\hline Number of subjects & 150 & 36 & \\
\hline Gender, female/male & $75 / 75$ & $12 / 24$ & 0.072 \\
\hline Median age, years & $50.0(49.1 \pm 12.4)$ & $55.0(54.1 \pm 18.2)$ & 0.120 \\
\hline Neutrophil & $7.8(8.6 \pm 4.8)$ & $4.4(4.4 \pm 1.0)$ & 0.000 \\
\hline Lymphocyte & $1.7(1.8 \pm 1.0)$ & $2.7(2.6 \pm 0.7)$ & 0.000 \\
\hline NLR & $4.7(7.0 \pm 9.3)$ & $1.7(1.8 \pm 0.6)$ & 0.000 \\
\hline WBC (K/mm $\left.{ }^{3}\right)$ & $11.0(11.6 \pm 5.2)$ & $7.7(8.0 \pm 1.9)$ & 0.000 \\
\hline ESR (mm/S) & $20.0(29.1 \pm 27.8)$ & $10.0(9.7 \pm 2.3)$ & 0.000 \\
\hline CRP (mg/L) & $26.0(70.9 \pm 100.0)$ & $2.0(2.6 \pm 2.0)$ & 0.000 \\
\hline $\begin{array}{l}\text { AP: Acute pancreatitis, WBC: White blood cells, CRP: C-reactive protein, ESR: } \\
\text { Erythrocyte sedimentation rate, NLR: Neutrophil-lymphocyte ratio }\end{array}$ \\
\hline
\end{tabular}


have 95\% discriminatory power with an optimal threshold value of 3 , sensitivity of $95.5 \%$ and specificity of $70 \%$ (Figure 1). When AP patients were evaluated in terms of etiology, mean NLR was $6.99 \pm 8.06$ in biliary AP and $6.94 \pm 9.78$ in non-biliary AP. There was no statistically significant difference between biliary and non-biliary AP in terms of NLR ( $p>0.05$, Table 2$)$. There was no statistically significant difference between biliary and non-biliary AP in terms of CRP, sedimentation, neutrophil and lymphocyte levels ( $p>0.005)$. According to the Ranson's criteria, mean

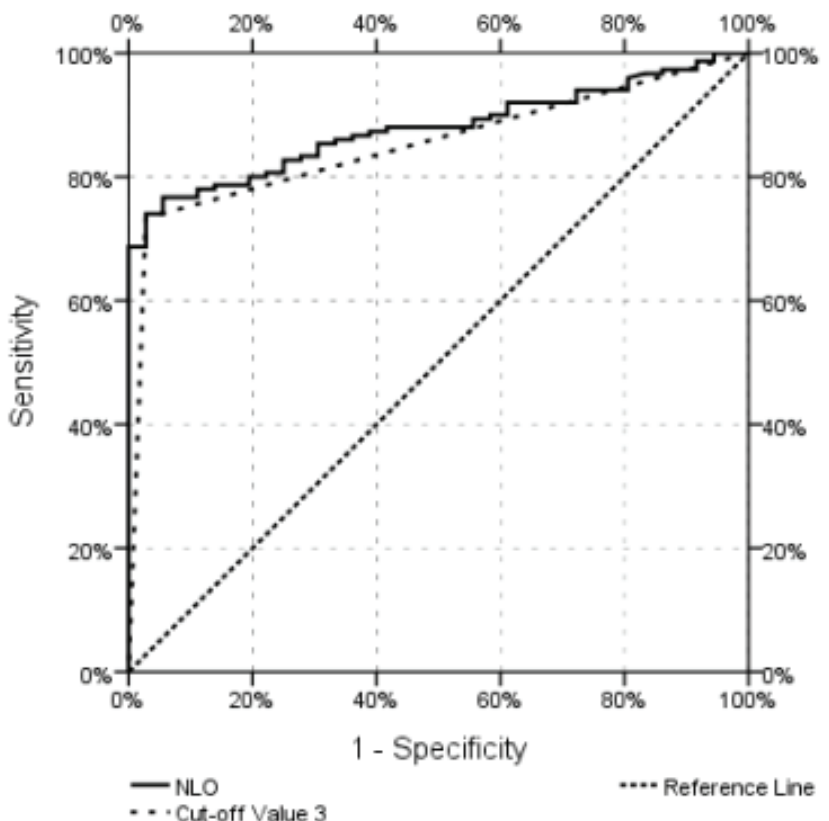

Figure 1. ROC curve analysis of neutrophil-lymphocyte ratio NLO: Neutrophil-lymphocyte ratio

\begin{tabular}{|c|c|c|c|}
\hline & $\mathbf{n}$ & NLR & $\mathbf{p}$ \\
\hline \multicolumn{4}{|l|}{ Etiology } \\
\hline Biliary & 61 & $6.99 \pm 8.06$ & 0.572 \\
\hline Non-biliary & 89 & $6.94 \pm 9.78$ & \\
\hline \multicolumn{4}{|l|}{ Ranson's criteria at 48 hours } \\
\hline $1-3$; mild pancreatitis & 121 & $6.26 \pm 9.72$ & $<0.01^{*}$ \\
\hline 4-11; severe pancreatitis & 29 & $9.85 \pm 6.70$ & \\
\hline \multicolumn{4}{|c|}{ Modified Glasgow Prognostic criteria (within 48 hours) } \\
\hline$<3$; mild pancreatitis & 123 & $5.90 \pm 8.76$ & $<0.01^{*}$ \\
\hline$\geq 3$; severe pancreatitis & 27 & $11.79 \pm 10.31$ & \\
\hline \multicolumn{4}{|l|}{ Balthazar score } \\
\hline $0-3$; mild pancreatitis & 73 & $5.80 \pm 4.24$ & 0.401 \\
\hline 4-6; moderate pancreatitis & 38 & $4.64 \pm 2.60$ & \\
\hline 7-10; severe pancreatitis & 17 & $7.29 \pm 5.31$ & \\
\hline
\end{tabular}

NLR was $9.85 \pm 6.70$ in patients with severe AP and $6.26 \pm 9.72$ in patients with mild AP. NLR was significantly higher in cases with severe AP $(p<0.01)$. According to mGPS, mean NLR was $11.79 \pm 10.31$ in patients with severe AP and $5.90 \pm 8.76$ in patents with mild AP. The difference between two groups was statistically significant $(p<0.05)$. According to the Ranson's criteria and mGPS, CRP levels in patients with severe AP were significantly higher than those with mild AP ( $p=0.003$ for Ranson's criteria and $p<0.05$ for mGPS). There was no difference between the groups in terms of sedimentation ( $p>0.005)$. According to Balthazar score, there was no statistically significant difference between NLR, sedimentation, neutrophil and lymphocyte levels $(p>0.05)$. However, CRP was highest in severe AP.

When the correlation between NLR and sedimentation, CRP, neutrophil and lymphocyte parameters in patients with AP was investigated, NLR was found to have positive correlation with CRP and neutrophil levels $(r=0.296, p<0.001 ; r=0.727$, $p<0.001$, respectively) and negative correlation with lymphocyte levels $(r=-0.625, p<0.001)$. There was no significant correlation between NLR and sedimentation $(r=0.126, p>0.17)$. There was no statistically significant difference between complicated and non-complicated cases in terms of NLR ( $p>0.05)$. There was also no statistically significant correlation between NLR and length of hospital stay ( $p>0.05)$.

\section{DISCUSSION}

AP is an inflammatory disease of the pancreas with very different clinical features, ranging from transient abdominal symptoms to death (7). The incidence of AP has increased in many European and Scandinavian countries due to increased alcohol consumption and increased diagnostic methods (13). Since it is sometimes not recognized or diagnosed after death, the actual incidence is unknown. But the incidence of AP is assumed to be $5-35 / 100.000$ (2). Recognition and evaluation of AP at an early stage is important in managing the treatment and avoiding recurrent episodes of pancreatitis. Gallstones and alcohol are the most common causes of AP. Other causes of AP include metabolic causes such as hyperlipidemia and hypercalcemia, surgical interventions, trauma, endoscopic retrograde cholangiopancreatography, infectious causes such as hepatitis B, cytomegalovirus and mycoplasma, some drugs, snake poisons or scorpion toxins (3). In our study, the etiology of AP was 41\% biliary and 59\% non-biliary (alcohol, hypertriglyceridemia, drugs, autoimmunity, trauma, idiopathic). In AP, there is still no easy, practical, inexpensive marker or parameter that will allow early diagnosis and give us an idea about the course and prognosis 
of the disease. This study investigated whether NLR could be used as a parameter in the diagnosis, severity, progression and follow-up of AP. Leukocytes are markers of infection and inflammation, and are used in prognosis and follow-up in a majority of inflammatory diseases. They are also the part of many prognostic scoring systems for AP, including Ranson's criteria, Glaskow-Imrie and APACHE II $(10,11,14)$.

Neutrophils are the most common type of leukocytes in the peripheral blood, which are produced from the stem cells in the bone marrow and then disseminated into circulation. Lymphocytes are specialized cells in the organism and carry various antigen receptors that recognize many foreign antigens. These receptors play an important role in response to these antigens and in the development of immune memory. Neutrophils spread inflammation and tissue destruction in AP through the activation of inflammatory cytokines [interleukin (IL)-6, IL-8 and tumor necrosis factor (TNF) alpha], a range of proteolytic enzymes (myeloperoxidase, elestase, collagenase) and free oxygen radicals. Lymphocytes increase following the initial stress, then fall within the first 24 hours and lymphopenia develops. Uncontrolled inflammation is considered to accelerate lymphopenia with accelerated apoptosis. Lymphopenia is associated with high mortality in patients with septic shock $(8,9)$. Pezzilli et al. (15) reported lymphopenia in patients with AP that continued on day 1 , followed by days 3 and 5 compared to other acute abdominal conditions and healthy controls. Consistent with the literature, this study found that patients with AP had a higher neutrophil level in the first 24 hours than the control group. However, lymphocyte level was significantly lower in AP group than in the control group (Table 1).

Nowadays, there are many studies in the literature showing the relationship between NLR and inflammation as well as various diseases. Some of the publications show that NLR can be used as an indicator of inflammation in various diseases such as ischemic stroke, atherosclerotic diseases, acute coronary syndrome and AP along with various types of cancer (16-19). Gibson et al. (20) stated that preoperative NLR accurately predicted the development of severe cholecystitis. Azab et al. (21) found that NLR increased in patients with AP. They also reported that NLR was superior to total WBC count or individual neutrophil and lymphocyte counts in predicting intensive care unit admission and mortality for AP. Our study supports the study by Azab et al. (21) and NLR was found to be significantly higher in AP group than in the control group. When the etiologic causes of AP were divided into two groups as biliary and non-biliary, there was no statistically significant difference between two groups in terms of NLR in our study $(p>0.05)$. In a study conducted by Kara et al. (22), NLR was found to be high in patients with AP. However, there was no statistically significant difference between non-biliary and biliary AP in terms of NLR. Suppiah et al. (16) reported that increased NLR in patients with AP could effectively differentiate between patients with mild and severe AP. In our study, NLR in the first 24 hours was significantly higher in patients with AP than in the control group. In addition, NLR in patients with mild and severe AP was significantly higher when stratified according to the Ranson's criteria and mGPS ( $p<0.05)$. In a study of patients with AP, NLR cut-off value for the severity of the disease was suggested to be above 4.7 (21). Our study found that a cut-off value of 3 for NLR had $95.5 \%$ sensitivity and $70 \%$ specificity for diagnosis of AP. In recent years, the focus has been on a wide variety of clinical parameters, single biochemical markers, scoring systems and imaging procedures for the prediction of severe AP. Gülen et al. (23) demonstrated that contrast-enhanced $\mathrm{CT}$ imaging might be effective in determining the diagnosis and prognosis of AP. CT results of the patients included in this study were also re-evaluated by a radiologist according to Balthazar score. However, there was no statistically significant difference in terms of NLR when the patients were classified according to Balthazar radiological criteria ( $p>0.05$ ). CRP is an acute phase reactant produced by the liver in response to IL-1, IL- 6 and TNF- $\alpha$, and is a low-cost marker that can be widely used to assess the severity of AP (24). A study conducted to predict AP showed that the simultaneous evaluation of erythrocyte sedimentation rate (ESR) and CRP is more successful than evaluating both variables separately. ESR can predict severe AP with slightly lower performance than that of CRP (25). In this study, we evaluated the relationship between ESR and CRP value and AP. We found no statistically significant difference in CRP levels between patients with biliary and non-biliary AP. According to Ranson's criteria and mGPS, CRP levels were significantly higher in patients with severe pancreatitis than in patients with mild pancreatitis. When CRP levels were examined according to Balthazar score, there was a difference between the grades. CRP levels were significantly higher in severe pancreatitis than mild pancreatitis. In addition, there was a statistically significant positive correlation between NLR and CRP, and these findings support the use of NLR as an inflammation marker in conjunction with CRP. Gürleyik et al. (26) reported that clinically severe AP had a longer hospital stay than that of clinically mild cases. In our study, no significant difference was found between severe and mild cases in terms of length of hospital stay. 


\section{CONCLUSION}

In conclusion, NLR elevation was higher in the patient group than in the control group. NLR level was high in patients with severe pancreatitis according to Ranson's criteria and mGPS, but no difference was found in patients with severe pancreatitis according to the Balthazar criteria that is based on CT scoring. From this point of view, NLR can be used to diagnose AP as it can be correlated with CRP, which is widely used in routine practice. It can also be considered as a practical test for the early prediction of severe pancreatitis according to the Ranson's criteria and mGPS.

\section{Ethics}

Ethics Committee Approval: The study was approved by the İstanbul Okmeydanı Training and Research Hospital Ethics Committee (approval no: 481, 17.05.2016).

Informed Consent: Retrospective study.

Peer-review: Externally peer-reviewed.

\section{Authorship Contributions}

Concept: Y.G., M.A., Design: Y.G., J.M., Data Collection or Processing: J.M., Analysis or Interpretation: Y.G., J.M., M.A., Literature Search: J.M., Writing: J.M., Y.G.

Conflict of Interest: No conflict of interest was declared by the authors.

Financial Disclosure: The authors declared that this study received no financial support.

\section{REFERENCES}

1. Vege SS. Clinical mnifestations and diagnosis of acute pankreatitis. Up to date, 2017.

2. Vege SS, Yadav D, Chari ST. Pancreatitis. In: Talley NJ, Locke GR, Saito YA editors. Gl epidemiology Malden, MA: Blackwell Publishing; 2007.

3. Cappell MS. Acute pancreatitis: etiology, clinical presentation, diagnosis, and therapy. Med Clin North Am 2008;92:889-923.

4. Lund H, Tønnesen H, Tønnesen MH, Olsen O. Long-term recurrence and death rates after acute pancreatitis. Scand J Gastroenterol 2006;41:234-8.

5. Windsor JA. Assessment of the severity of acute pancreatitis: no room for complacency. Pancreatology 2008;8:105-9.

6. Zahorec R. Ratio of neutrophil to lymphocyte counts-rapid and simple parameter of systemic inflammation and stress in critically ill. Bratisl Lek Listy 2001;102:5-14.

7. Otsuki M, Takeda K, Matsuno S, Kihara Y, Koizumi M, Hirota M, et al. Criteria for the diagnosis and severity stratification of acute pancreatitis. World J Gastroenterol 2013;19:5798-805.

8. de Jager CP, van Wijk PT, Mathoera RB, de Jongh-Leuvenink J, van der Poll T, Wever PC. Lymphocytopenia and neutrophillymphocyte count ratio predict bacteremia better than conventional infection markers in an emergency care unit. Crit Care 2010;14:R192.
9. Le Tulzo Y, Pangault C, Gacouin A, Guilloux V, Tribut O, Amiot L, et al. Early circulating lymphocyte apoptosis in human septic shock is associated with poor outcome. Shock 2002;18:487-94.

10. Corfield A, Cooper MJ, Williamson R, Mayer AD, McMahon M, Dickson AP, et al. Prediction of severity in acute pancreatitis: Prospective comparison of three prognostic indices. Lancet 1985;2:403-7.

11. Demmy TL, Burch JM, Feliciano DV, Mattox KL, Jordan GL Jr. Comparison of multipl parameter prognostics systems in acute pancreatitis. Am J Surg 1988;156:2-6.

12. Balthazar EJ, Robinson DL, Megibow AJ, Ranson JH. Acute pancreatitis: value of CT in establishing prognosis. Radiology 1990;174:331-6.

13. Toouli J, Brooke-Smith M, Bassi C, Carr-Locke D, Telford J, Freeny P, et al. Guidelines for the management of acute pancreatitis. J Gastroenterol Hepatol 2002;Suppl:S15-39.

14. Knaus WA, Draper Ea, Wagner DP, Zimmeran JE. APACHE II: a severity of disease classification system. Crit Care Med 1985;13:818-29.

15. Pezzilli R, Billi P, Beltrandi E, Casadei Maldini M, Mancini R. Impaired lymphocyte proliferation in human acute pancreatitis. Digestion 1997;58:431-6.

16. Suppiah A, Malde D, Arab T, Hamed M, Allgar V, Smith AM, et al. The prognostic value of the neutrophil-lymphocyte ratio (NLR) in acutepancreatitis: identification of an optimal NLR. J Gastrointest Surg 2013;17:675-81.

17. Binnetoğlu E, Akbal E, Güneş F, Sen H. The prognostic Value of Neutrophillymphosyte Ratio in Acute Pancreatitis is Controversial.J Gastrointest Surg 2014;18:885.

18. Gökhan S, Özhasenekler A, Mansur Durgun H, Akil E, Ustündag M, Orak M. Neutrophil lymphocyte ratios in stroke subtypes and transient ischemic attack. Eur Rev Med Pharmacol Sci 2013;17:653-7.

19. Temiz A, Gazi E, Güngör Ö, Barutçu A, Altun B, Bekler A, et al. Platelet/ lymphocyte ratio and risk of in-hospital mortality in patients with STelevated myocardial infarction. Med Sci Monit 2014;20:660-5.

20. Gibson PH, Croal BL, Cuthbertson BH, Small GR, Ifezulike Al, Gibson G, et al. Preoperative neutrophil-lymphocyte ratio and outcome from coronary artery bypass grafting. Am Heart J 2007;154:995-1002.

21. Azab B, Jaglall N, Atallah JP, Lamet A, Raja-Surya V, Farah B, et al. Neutrophillymphocyte ratio as a predictor of adverse outcomes of acute pancreatitis. Pancreatology 2011;11:445-52.

22. Kara H, Doğru A, Değirmenci S. Bayır A. Ak A. Kafalı ME, et al. Diagnostic value of neutrophil-to-lymphocyte ratio in emergency department patients diagnosed with acute pancreatitis. Çukurova Med J 2016:41:55-60.

23. Gülen B, Sonmez E, Yaylaci S, Serinken M, Eken C, Dur A, et al. Effect of harmless acute pancreatitis score, red cell distribution width and neutrophil/ lymphocyte ratio on the mortality of patients with nontraumatic acute pancreatitis at the emergency department. World J Emerg Med 2015;6:2933.

24. Khanna AK, Meher S, Prakash S, Tiwary SK, Singh U, Srivastava A, et al. Comparison of Ranson, Glasgow, MOSS, SIRS, BISAP, APACHE-II, CTSI Scores, IL-6, CRP, and Procalcitonin in Predicting Severity, Organ Failure, Pancreatic Necrosis, and Mortality in Acute Pancreatitis. HPB Surg 2013;1-10.

25. Lankisch PG, Weber-Dany B, Hebel K, Maisonneuve P, Lowenfels AB. The harmless acute pancreatitis score: a clinical algorithm for rapid initial stratification of nonsevere disease. Clin Gastroenterol Hepatol 2009;7:702-5.

26. Gürleyik G, Emir S, Kiliçoglu G, Arman A, Saglam A. Computed tomography severity index, APACHE II score, and serum CRP concentration for predicting the severity of acute pancreatitis. JOP 2005;6:562-7. 